\title{
BMJ Open Measurement properties of self-report physical activity assessment tools in stroke: a protocol for a systematic review
}

\author{
Júlia Caetano Martins, ${ }^{1}$ Larissa Tavares Aguiar, ${ }^{1}$ Sylvie Nadeau, ${ }^{2}$ \\ Aline Alvim Scianni, ${ }^{1}$ Luci Fuscaldi Teixeira-Salmela, ${ }^{1}$ \\ Christina Danielli Coelho de Morais Faria ${ }^{1}$
}

To cite: Martins JC, Aguiar LT, Nadeau S, et al. Measurement properties of self-report physical activity assessment tools in stroke: a protocol for a systematic review. BMJ Open 2017;7: e012655. doi:10.1136/ bmjopen-2016-012655

- Prepublication history and additional material is available. To view please visit the journal (http://dx.doi.org/ 10.1136/bmjopen-2016012655).

Received 15 May 2016 Revised 11 August 2016 Accepted 7 October 2016

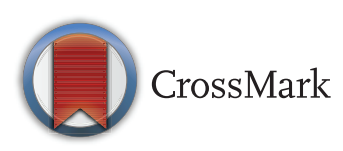

${ }^{1}$ Department of Physical Therapy, Universidade Federal de Minas Gerais (UFMG), Belo Horizonte, Brazil ${ }^{2}$ Université de Montreal (UdeM), Centre de recherche interdisciplinaire en réadaptation (CRIR), Institut de réadaptation GingrasLindsay de Montréal (IRGLM), CIUSSS CentreSud-de-l'Île-de-Montréal, Montréal, Québec, Canada

Correspondence to Dr Christina Danielli Coelho de Morais Faria; cdcmf@ufmg.br

\section{ABSTRACT}

Introduction: Self-report physical activity assessment tools are commonly used for the evaluation of physical activity levels in individuals with stroke. A great variety of these tools have been developed and widely used in recent years, which justify the need to examine their measurement properties and clinical utility. Therefore, the main objectives of this systematic review are to examine the measurement properties and clinical utility of self-report measures of physical activity and discuss the strengths and limitations of the identified tools.

Methods and analysis: A systematic review of studies that investigated the measurement properties and/or clinical utility of self-report physical activity assessment tools in stroke will be conducted. Electronic searches will be performed in five databases: Medical Literature Analysis and Retrieval System Online (MEDLINE) (PubMed), Excerpta Medica Database (EMBASE), Physiotherapy Evidence Database (PEDro), Literatura Latino-Americana e do Caribe em Ciências da Saúde (LILACS) and Scientific Electronic Library Online (SciELO), followed by hand searches of the reference lists of the included studies. Two independent reviewers will screen all retrieve titles, abstracts, and full texts, according to the inclusion criteria and will also extract the data. A third reviewer will be referred to solve any disagreement. A descriptive summary of the included studies will contain the design, participants, as well as the characteristics, measurement properties, and clinical utility of the self-report tools. The methodological quality of the studies will be evaluated using the COnsensus-based Standards for the selection of health Measurement INstruments (COSMIN) checklist and the clinical utility of the identified tools will be assessed considering predefined criteria. This systematic review will follow the Preferred Reporting Items for Systematic Review and Meta-Analyses (PRISMA) statement.

Discussion: This systematic review will provide an extensive review of the measurement properties and clinical utility of self-report physical activity assessment tools used in individuals with stroke, which would benefit clinicians and researchers.

Trial registration number: PROSPERO CRD42016037146.

\section{Strengths and limitations of this study}

- This systematic review will include all self-report physical activity assessment tools used with individuals with stroke published in English, Spanish, French and Portuguese.

- This systematic review protocol will be reported in accordance with the Preferred Reporting Items for Systematic review and Meta-Analyses Protocols (PRISMA-P).

- The methodological quality of the included studies will be assessed by the cOnsensus-based Standards for the selection of health Measurement INstruments (COSMIN) guidelines.

- The clinical utility will be assessed to quantify the practicalities of using self-report physical activity assessment tools in individuals with stroke.

- This systematic review will not include other neurological populations, besides stroke.

\section{INTRODUCTION}

The maintenance of adequate levels of physical activity by individuals with stroke may reduce the recurrence of stroke and other cardiovascular diseases, as well as prevent secondary disabilities. ${ }^{1}$ The use of appropriate measures of physical activity is important for determining trends in physical activity levels over time, the effects of interventions aimed at increasing physical activity levels, and the health benefits of regular practice of physical activity. $^{2}$

Measures of physical activity assess the actual or perceived ability of the individuals to carry out a variety of daily tasks and recreational or competitive sports. ${ }^{2}$ However, the multidimensional and individual-specific nature of physical activity have resulted in the development of several measures, contributing to the lack of consensus from clinicians and researchers regarding the best measure to be used. ${ }^{3}$ 
Direct measures of physical activity levels have been used to provide information on intensity, volume, duration of practice of physical activity, as well as the distance travelled and energy expenditure. ${ }^{4} 5$ These measures include calorimetry, physiological markers, motion sensors, activity monitors and direct observations. ${ }^{5}$ Direct measures of physical activity provide more accurate assessments of physiological or mechanical parameters that correspond to the physical activity levels, when compared with self-report measures. ${ }^{6}{ }^{7}$ However, direct measures of physical activity are expensive and require time and specialised training, making them difficult to be applied within clinical settings and in large epidemiologic studies. ${ }^{5}$

Self-report measures of physical activity have been used for nearly 50 years, to determine the frequency, duration, intensity, and type of physical activity in various populations and health conditions. ${ }^{8}$ These consist of questionnaires, diaries/logs, surveys, and interviews. ${ }^{5}$ The main limitation of these measures is that individuals may overestimate or underestimate their true energy expenditure and rates of inactivity. ${ }^{59}{ }^{10}$ However, they are frequently used, since they have low cost, require lower burden of the participants, many are easy to administer, have the potential to inform about different types of activities performed in a variety of contexts, have general acceptance within clinical settings and large epidemiologic studies, and offer closer comparisons with normative data, which is often collected by self-report measures. ${ }^{5} 11$

The ideal tool for the assessment of physical activity should be responsive to changes in physical activity levels, easy to use, and provide accurate estimations of intensity, volume, duration and frequency of the performed activity. ${ }^{6}$ The appropriate method for measuring physical activity at various levels depends on factors, such as the number of individuals to be monitored, the time required to apply the tool, the purpose and setting in which it will be applied, and the available finances. ${ }^{6} 12$

Most self-report tools were designed to measure multiple dimensions of physical activity, such as type, location, domain, and context of the activity. ${ }^{13}$ They also provide estimations of the time spent in activities of various levels of intensity, and are able to rank the individuals, according to the intensity levels of the reported activity. ${ }^{13}$ The wide use of these measures justifies the need to identify their measurement properties and clinical utility.

In 2010, a systematic review ${ }^{2}$ assessed the measurement properties of self-report physical activity questionnaires in healthy individuals aged 18-55 years. The authors included only studies published in English and excluded other types of self-report measures, such as diaries/logs, surveys, and interviews. They were not able to identify the best self-reported tool, probably due the heterogeneity and ambiguity in terminology, which made synthesis difficult.
In 2015, another systematic review ${ }^{3}$ evaluated the measurement properties of self-reported physical activity questionnaires in healthy adults aged 18-60 years. The authors included only studies published in English and excluded self-report measures obtained via telephone, diaries/logs tools and long-term recalls of physical activity (>6 months). The results were inconclusive regarding the best self-report tool, probably due to ambiguities in terminology, inconsistencies within the types of assessed physical activity, limited measurement property investigation, and poor methodological quality of various studies.

In 2015, a systematic review, aimed at describing how physical activity was monitored following stroke using direct physical activity assessment tools, as well as reporting the measurement properties of these tools, was published. ${ }^{14}$ The results showed that an ideal single tool for clinical application in people with stroke could not be identified. However, the authors emphasised that several tools provided comprehensive assessment data. In addition, they suggested that test-retest reliability of several direct physical activity assessment tools need to be established for the stroke population. It is important to point out that studies that used self-report physical activity assessment tools and were not published in English, were excluded from the review.

It is important to establish the measurement properties of self-report physical activity assessment tools for individuals with stroke, since they often show low levels of physical activity, which is an important risk factor for the recurrence of stroke and secondary disabilities, and also a possible limitation to their participation in some physical activities and research studies, mainly the ones that involve various assessments and follow-ups.

There are various self-report tools described in the literature for the assessment of physical activity levels of individuals with stroke. However, no systematic review, that gathered information on the measurement properties or clinical utility of these tools, was found. Therefore, the main objectives of this systematic review are to examine the measurement properties and clinical utility of self-report measures of physical activity and discuss the strengths and limitations of the identified tools.

\section{METHODS}

\section{Study design and registration}

This systematic review was registered in the International Prospective Register of Systematic Reviews (PROSPERO) on April 1st, 2016 (\#CRD42016037146; http://www.crd. york.ac.uk/PROSPERO/). The protocol was written and reported using the Preferred Reporting Items for Systematic Reviews and Meta-Analyses Protocols (PRISMA-P) statement and the PRISMA-P checklist (see research checklist). ${ }^{15}$

\section{Eligible studies}

All full-text papers that investigated the measurement properties or clinical utility of self-report measures of 
physical activity levels in individuals with stroke will be included, being physical activity defined as any bodily movement produced by skeletal muscles, that requires energy expenditure. ${ }^{16}$ There will be included self-report measures of all types of physical activity (incidental or planned activities) performed at various intensities (light, moderate or vigorous). Studies reporting sedentary behaviour, that is, in accordance with the previous definition of physical activity, will also be included.

All studies published in English, Spanish, French and Portuguese will be included. Studies reporting only walking or exercise capacity, gait patterns, or ability to perform activities of daily living (eg, Barthel index or functional independence measure), which are not measures of physical activity levels, will be excluded. Systematic reviews will also be excluded, but their reference lists will be screened for relevant studies.

All studies with adults ( $\geq 18$ years of age), who had a stroke, will be included, without further restrictions. Studies with mixed groups will be excluded. In case it is not clear that the study met the inclusion criteria, the correspondent author will be contacted.

\section{Search strategy for identification of relevant studies}

A comprehensive search will be carried out for articles indexed on MEDLINE (via PubMed), Excerpta Medica Database (EMBASE), Physiotherapy Evidence Database (PEDro), Literatura Latino-Americana $e$ do Caribe em Ciências da Saúde (LILACS), and Scientific Electronic Library Online (SciELO) databases. Hand searches of the reference lists of the included studies or of relevant reviews will be employed. A search strategy was designed with the assistance of an experienced researcher and previous published search strategy with related terms. ${ }^{4}{ }^{14}{ }^{17-19}$ The established search strategy for the MEDLINE database (see online supplementary appendix 1) was adapted to the other databases. The search strategy contains blocks of search terms related to the following aspects:

1. Target population: stroke. The search strategy was based on a Cochrane systematic review developed by Barclay et al. ${ }^{17}$

2. Outcome measure: physical activity. The search strategy was based on two previously published systematic reviews on direct measures of physical activity levels. ${ }^{14} 18$

3. Measurement properties: the search strategy was based on a systematic review developed by Silva et al. ${ }^{19}$

4. Instruments: self-report measures. The search strategy was developed based on a guide for the assessment of physical activity levels developed by the American Heart Association. ${ }^{4}$

\section{Screening of the studies}

The electronic searches will be saved and maintained in a file, to record the initial search strategy and subsequent modifications, the searched databases and the details of the identified studies. Duplicate articles will be removed, as the same article may be located in more than one database. If there is more than one article from the same study, different data may be extracted from different articles, where relevant. The authors will be contacted, when necessary, for additional information.

One reviewer (JCM) will include the search strategy in all databases and extract the titles and abstracts for analyses. Then, two reviewers (JCM and LTA) will independently assess the titles and abstracts of all identified studies and exclude those that do not meet the inclusion criteria. The full text of the remaining studies will be independently reviewed by the same authors (JCM and LTA) for eligibility screening, using a standardised eligibility criteria sheet. Disagreements will be resolved by discussion and consensus. If required, a third reviewer (CDCMF) will be consulted. A study selection file will be maintained, to record the references for the excluded studies and the reason for excluding them. Following the PRISMA guidelines,${ }^{20}$ a diagram will be created to report the flow through the study.

\section{Data extraction}

Relevant data from all the included studies will be summarised in tables. Data from all the included articles will be independently extracted by two reviewers (JCM and LTA), who will work in pairs on defined sets of articles. Disagreements will be resolved by discussion and consensus.

Evidence tables will include the following data: reference, name of the self-report physical activity assessment tool, target population to whom the tool was originally developed, geographical location, language, setting, study type, main characteristics of the participants (such as age, sex, type and time since the onset of the stroke, severity of the stroke, and possible cognitive impairment), assessed physical activity domains, number of items and subscales, number and type of response categories, recall period, scoring algorithm, time needed for administration, mode of administration (self-administered or interview), how a full copy of the tool can be obtained, the instructions given to apply the tool, the available versions and translations, the investigated measurement properties and/or clinical utility, as well as the obtained results. Different language versions of the same tool will be separately verified throughout the review. ${ }^{21}$ The main reason for doing so is because it is problematic to assume that different language versions of measurement instruments exhibit the same measurement properties. ${ }^{21}$ If general characteristics of the self-report measures cannot be extracted from the included studies, the original paper will be consulted to obtain the missing information. ${ }^{21}$

\section{Content comparison}

An overview of all self-report measures of physical activity levels on the content domain level will be presented, to visualise the content covered by the different measures. The original development paper will be consulted 
to obtain this information. The terminologies and definitions of the types of the assessed physical activity will be standardised, based on previously published guidelines. ${ }^{4}$

\section{Assessment of the methodological quality of the included studies}

The COnsensus-based Standards for the selection of health Measurement INstruments (COSMIN) checklist $^{22} 23$ will be used to evaluate the methodological quality of the included studies. In the COSMIN checklist (http://www.cosmin.nl), the following four domains are distinguished: reliability, validity, responsiveness, and interpretability. ${ }^{23}$ For all measurement properties, the COSMIN checklist consists of five to 18 items, which cover the methodological standards (organised in nine boxes for the nine measurement properties). In addition, each item can be scored on a 4-point rating scale ('poor,' 'fair,' 'good,' 'excellent'). By taking the lowest rating for each item in one box, an overall quality score ('poor,' 'fair,' 'good,' 'excellent') is separately obtained for each measurement property. ${ }^{24}$ This is essential to prevent the risk of selecting tools, which were developed using designs with poor methodological rigor. ${ }^{25}$ The quality criteria for the investigation of measurement properties proposed by Terwee $e t a l,{ }^{26}$ will also be used. Therefore, the measurement properties reported by the included studies will be rated as positive, negative, or questionable, following those criteria. An ideal tool should have positive ratings. ${ }^{26}$

\section{Assessment of the clinical utility of the identified tools}

Data on interpretability and clinical utility (feasibility) will be collected, where reported. Furhtermore, the clinical utility will be assessed to quantify the practicalities of the identified tool. Thus, previously recommended criteria will be applied, based on the following factors that could influence the clinicians' decisions of using a measurement tool in their clinical practice: $:^{27} 28$

1. Time taken to administer, analyse and interpret the measures: $<10 \mathrm{~min}$ (3 points); $10-30 \mathrm{~min}$ (2 points); 30-60 min (1 point) and $>1 \mathrm{~h}$ (0 point).

2. Cost: $3=<£ 100 ; 2=£ 100-500 ; 1=£ 500-1000 ; 0>£ 1000$.

3 . Does the measurement tool need specialised equipment and training to use? $2=$ no; $1=$ yes, but simple and clinically feasible; $0=$ yes and not clinically feasible/unknown.

4. Is the measurement tool portable? Can it be taken to the patient? $2=$ yes, easily (can fit into the pocket); $1=$ yes (can fit in a briefcase or trolley); $0=$ no or very difficult.

5. Is the measurement tool accessible? Can a detailed instruction for application be obtained? $2=$ yes (full standardised operating procedure/instruction manual can be obtained from the article or a website); $1=$ no, but operation can be simply worked out from a description in the article; $0=$ no operating instructions available.
The score on each criteria, as well as the total score (maximum of 12 points) will also be reported in a table. Tools with a total score $<10$ points are not considered feasible for clinical use and this criterion will be applied in the present study. ${ }^{28}$

\section{Data synthesis}

A systematic narrative synthesis will be provided in text and table formats, to summarise and discuss the sample and methodological characteristics, and findings regarding the measurement properties or clinical utility of the included studies on self-report measures of physical activity levels in individuals with stroke. If a self-report measure is evaluated in several studies and the findings conflict each other, all the results will be presented and some possible hypothesis for the different results will be raised and discussed.

\section{DISCUSSION}

This review will examine the measurement properties and clinical utility of self-report physical activity assessment tools for individuals with stroke. The purpose is to provide a discussion of the strengths and limitations of the different tools used for the assessment of physical activity levels in individuals with stroke, by analysing the methodological quality of the included studies, as well as the clinical utility of the identified tools.

Physical inactivity is a significant cause of global morbidity and mortality. Individuals with stroke demonstrate low levels of physical activity, which increase the risks for further cardiovascular and stroke-related diseases and disabilities. Therefore, it is important to assess the physical activity levels of this population with appropriate measures. Adequate measurement tools provide valid and reliable results, which may allow therapists to establish interventions to increase physical activity levels of individuals with stroke.

The choice of self-report tools which have adequate measurement properties, for the assessment of physical activity levels is important, given the variety of measures that have been developed to measure this multidimensional construct. This is especially important for clinical use, as there is a need to screen individuals with stroke at low levels of physical activity. The identification and use of self-report measures of physical activity for the population with stroke, that have appropriate measurement properties and clinical utility may also enhance the credibility and comparability of interventions aiming at improving physical activity levels.

It is possible that a combination of self-report and direct (objective) measures of physical activity would be the best option to obtain a comprehensive evaluation of the subjects' physical activity levels. Considering that a systematic review was recently published regarding how physical activity was monitored following stroke using direct physical activity assessment tools, as well as their measurement properties, ${ }^{14}$ the results provided by the 
present study, associated with those of this previous systematic review, ${ }^{14}$ will facilitate the decision-making regarding the possible combination of these tools.

This review is transparent on its adherence to validated methods and employs a systematic and replicable approach regarding the searching, screening, appraising and extracting data from the current evidence base. The inclusion and exclusion criteria were well established and the search strategy is comprehensive. The involvement of two reviewers in screening, extracting data, and appraising the methodological quality of the included studies will enhance the credibility of the conclusions. The findings from this systematic review will be disseminated by scientific peer-reviewed publication and conference presentations.

Contributors JCM contributed to conception and design, data collection analysis and interpretation, manuscript writing, and final approval of the manuscript. LTA contributed to conception and design, data collection, analysis and interpretation, critical revision, and final approval of the manuscript. SN contributed to conception and design, data analysis and interpretation, critical revision, and final approval of the manuscript. AAS contributed to conception and design, data analysis and interpretation, critical revision and final approval of the manuscript. LFT-S contributed to conception and design, data analysis and interpretation, critical revision, and final approval of the manuscript. CDCMF contributed to conception and design, data analysis and interpretation, critical revision, and final approval of the manuscript. All authors read and approved the final manuscript. The guarantor of this study is CDCMF. Protocol amendments will be documented with the date of all amendments and with a description of the change and the rationale.

Funding Financial support for this research was provided by the CAPES (Coordenação de Aperfeiçoamento de Pessoal de Nível Superior), FAPEMIG (Fundação de Amparo à Pesquisa do Estado de Minas Gerais), CNPq (Conselho Nacional de Desenvolvimento Científico e Tecnológico) and PRPq/ UFMG (Pró-reitoria de Pesquisa da Universidade Federal de Minas Gerais). This financial support includes scholarships and research grants. CAPES, FAPEMIG, CNPq and PRPq/UFMG are not involved in any other aspect of this study protocol.

Competing interests None declared.

Provenance and peer review Not commissioned; externally peer reviewed.

Data sharing statement The findings from this systematic review will be disseminated by scientific peer-reviewed publication and conference presentations.

Open Access This is an Open Access article distributed in accordance with the Creative Commons Attribution Non Commercial (CC BY-NC 4.0) license, which permits others to distribute, remix, adapt, build upon this work noncommercially, and license their derivative works on different terms, provided the original work is properly cited and the use is non-commercial. See: http:// creativecommons.org/licenses/by-nc/4.0/

\section{REFERENCES}

1. Gallanagh S, Quinn TJ, Alexander J, et al. Physical activity in the prevention and treatment of stroke. ISRN Neurol 2011;2011:953818.

2. Van Poppel MN, Chinapaw MJ, Mokkink LB, et al. Physical activity questionnaires for adults: a systematic review of measurement properties. Sports Med 2010;40:565-600.

3. Silsbury Z, Goldsmith R, Rushton A. Systematic review of the measurement properties of self-report physical activity questionnaires in healthy adult populations. BMJ Open 2015;5: e008430.

4. Strath SJ, Kaminsky LA, Ainsworth BE, et al. Guide to the assessment of physical activity: clinical and research applications: a scientific statement from the American Heart Association. Circulation 2013;128:2259-79.

5. Prince SA, Adamo KB, Hamel ME, et al. A comparison of direct versus self-report measures for assessing physical activity in adults: a systematic review. Int J Behav Nutr Phys Act 2008;5:56.

6. Ainsworth B, Cahalin L, Buman M, et al. The current state of physical activity assessment tools. Prog Cardiovasc Dis 2015;57:387-95.

7. Westerterp KR. Assessment of physical activity: a critical appraisal. Eur J Appl Physiol 2009;105:823-8.

8. Epstein L, Miller GJ, Stitt FW, et al. Vigorous exercise in leisure time, coronary risk-factors, and resting electrocardiogram in middle-aged male civil servants. Br Heart J 1976;38:403-9.

9. Jobe JB, Mingay DJ. Cognitive research improves questionnaires. Am J Public Health 1989;79:1053-5.

10. Durante $\mathrm{R}$, Ainsworth BE. The recall of physical activity: using a cognitive model of the question-answering process. Med Sci Sports Exerc 1996;28:1282-91.

11. Helmerhorst $\mathrm{HJ}$, Brage $\mathrm{S}$, Warren J, et al. A systematic review of reliability and objective criterion related validity of physical activity questionnaires. Int J Behav Nutr Phys Act 2012;9:103.

12. Ainslie PN, Reilly T, Westerterp KR. Estimating human energy expenditure: a review of techniques with particular references to doubly labelled water 38. Sports Med 2003;33:683-98.

13. Warren JM, Ekelund $\mathrm{U}$, Besson $\mathrm{H}$, et al. Assessment of physical activity - a review of methodologies with reference to epidemiological research: a report of the exercise physiology section of the European Association of Cardiovascular Prevention and Rehabilitation. Eur J Cardiovasc Prev Rehabil 2010;17:127-39.

14. Fini NA, Holland $\mathrm{AE}$, Keating J, et al. How is physical activity monitored in people following stroke? Disabil Rehabil 2015;37: 1717-31.

15. Shamseer L, Moher D, Clarke M, et al. Preferred reporting items for systematic review and meta-analysis protocols (PRISMA-P) 2015: elaboration and explanation. BMJ 2015;349:g7647.

16. Caspersen CJ, Powell KE, Christenson GM. Physical activity exercise and physical fitness: definitions and distinctions for health-related research. Public Health Rep 1985;100:126-35.

17. Barclay RE, Stevenson TJ, Poluha W, et al. Interventions for improving community ambulation in individuals with stroke. Cochrane Database Syst Rev 2015;3:CD010200.

18. de Vries HJ, Kooiman TJ, van Ittersum MW, et al. Do activity monitors increase physical activity in adults with overweight or obesity? A systematic review and meta-analysis. Obesity (Silver Spring) 2016;24:2078-91.

19. Silva PFS, Quintino LF, Franco J, et al. Measurement properties and feasibility of clinical tests to assess sit-to-stand/ stand-to-sit tasks in subjects with neurological disease: a systematic review. Braz J Phys Ther 2014;18:99-110.

20. Moher D, Liberati A, Tetzlaff J, et al. Preferred reporting items for systematic reviews and meta-analyses: the PRISMA statement. PLoS Med 2009;6:e1000097.

21. Heinl D, Prinsen CA, Drucker AM, et al. Measurement properties of quality of life measurement instruments for infants children and adolescents with eczema: protocol for systematic review. Syst Rev 2016;5:25.

22. Mokkink LB, Terwee CB, Knol DL, et al. The COSMIN checklist for evaluating the methodological quality of studies on measurement properties: a clarification of its content. BMC Med Res Methodol 2010;10:22.

23. Mokkink LB, Terwee CB, Patrick DL, et al. The COSMIN study reached international consensus on taxonomy, terminology, and definitions of measurement properties for health-related patient reported outcomes. J Clin Epidemiol 2010;63:737-45.

24. Terwee CB, Mokkink LB, Knol DL, et al. Rating the methodological quality in systematic reviews of studies on measurement properties: a scoring system for the COSMIN checklist. Qual Life Res 2012;21:651-7.

25. Mokkink LB, Terwee CB, Gibbons E, et al. Inter-rater agreement and reliability of the COSMIN (COnsensus-based Standards for the selection of health status Measurement Instruments) checklist. BMC Med Res Methodol 2010;10:82.

26. Terwee CB, Bot SDM, de Boer MR, et al. Quality criteria were proposed for measurement properties of health status questionnaires. J Clin Epidemiol 2007;60:34-42.

27. Tyson S, Watson A, Moss S, et al. Development of an evidence based framework for the physiotherapy assessment of neurological conditions? Disab Rehabil 2007;30:142-4.

28. Tyson SF, Brown P. How to measure fatigue in neurological conditions? A systematic review of psychometric properties and clinical utility of measures used so far. Clin Rehabil 2014;28:804-16. 